\title{
Analysis of upper arm muscle activation using surface electromyography signals during drum playing
}

\author{
Hyun Ju Chong' ${ }^{1}$, Chun-Ki Kwon², Hyun-Joo Kang ${ }^{3}$, Soo Ji Kim,** \\ 'Department of Music Therapy, Ewha Womans University, Seoul, Korea \\ 2Department of Medical IT Engineering, Soonchunhyang University, Asan, Korea \\ ${ }^{3}$ Department of Sports Medicine, Soonchunhyang University, Asan, Korea \\ ${ }^{4}$ Department of Music Therapy Education, Ewha Womans University, Seoul, Korea
}

This study measured surface electromyography of the biceps brachii and triceps brachii during repeated drum playing with and without a drumstick to better understand activation of the upper arm muscles and inform the use of instrument playing for motor rehabilitation. A total of 40 healthy college students participated in this study. All participants were asked to strike a drum with their hand and with a drumstick at three different levels of stroke: soft, medium, and strong. The stroke order was randomly assigned to participants. A sound level meter was used to record the intensity of the drum playing. Surface electromyography signals were recorded at every hit during drum playing both with and without the drumstick in each of the three stroke conditions. The results demonstrated that the highest muscle activation was observed in both biceps brachii and triceps brachii with strong drum playing with and without the drumstick. A two-way repeated measures analysis of variance showed that there was a significant main effect for stroke intensity in muscle activation and produced sound level. While higher activation of the triceps brachii was observed for drum playing without a drumstick, no significant differences were found between the biceps brachii and sound level. This study demonstrated via surface electromyography data that greater muscle activation of the biceps brachii and triceps brachii does not occur with the use of drumsticks in drum playing. With the drum sound controlled, drum playing by hand can be an effective therapeutic intervention for the upper arm muscles.

Keywords: Surface electromyography, Upper extremity, Drum playing, Decibel

\section{INTRODUCTION}

Instrument playing as a system of sound production has received much attention in physical rehabilitation because it involves patterned physical movement. Instrument playing is highly effective as a therapeutic modality in rehabilitation because it induces repetitive physical movement in a predictable manner (Jeong, 2013). Drums are one of the easiest instruments to play because they involve simply striking the surface of the drum to make a sound. However, much of the research regarding instrument playing and muscle movement focuses on orchestra instruments and professional musicians. For example, researchers investigated muscle activation in the finger, wrist, and arm while playing string instruments (Guettler, 1992; Thiem et al., 1994) and keyboards (Lai et al., 2008; Oikawa et al., 2011; Penn et al., 1999). Findings from these studies confirmed the specific muscle groups that are involved in instrument playing by musicians who complete long-term music training. A positive relationship between forearm muscle activation and sound level for piano playing was revealed by surface electromyography (sEMG) data (Oikawa et al., 2011). With regards to different positions, the maximal voluntary contraction (MVC) was smallest at the neutral position, especially playing at a medium or strong sound level, indicating some degree of interaction between piano playing position and sound level.

With regard to EMG responses during percussive instrument playing, Fujii et al. (2009) examined wrist muscle activity underlying the stability of drumming. Participants with different music
${ }^{*}$ Corresponding author: Soo Ji Kim (iD http://orcid.org/0000-0002-7279-1804 Department of Music Therapy Education, Ewha Womans University, 209 Case Hall, 52 Ewhayeodae-gil, Seodaemun-gu, Seoul 03760, Korea Tel: +82-2-3277-6916, Fax +82-2-3277-6918, E-mail: specare@ewha.ac.kr Received: February 5, 2016 / Accepted: May 14, 2016
This is an Open Access article distributed under the terms of the Creative Commons Attribution Non-Commercial License (http://creativecommons.org/licenses/by-nc/4.0/) which permits unrestricted non-commercial use, distribution, and reproduction in any medium, provided the original work is properly cited. 
training backgrounds played the drum using dominant and nondominant hands separately to observe wrist flexion and extension at the flexor capri ulnaris and extensor carpi radialis. The results showed that the rate and evenness of inter-tap-interval in drumming increased with the level of drumming expertise. Further, cocontraction of EMG patterns between flexor capri ulnaris and extensor carpi radialis was found, indicating reciprocal wrist muscle activity underlying fast and stable drumming performance. Fujisawa and Miura (2010) examined how wrist muscle activity differed by music expertise, playing strategy, and drumstick size. Participants with various levels of drumming experience (i.e., nondrummer, novice drummer, amateur drummer, and trained drummer) played drums using drumsticks of varying weights and lengths. In general, the EMG activity measured at the flexor carpi ulnaris muscle of both arms was significantly less when participants engaged in free drum playing with a regular sized drumstick. The EMG activity was significantly greater with decreased length as well as with increased weight of the drumstick. Specific to the trained drummers, the amplitude of sEMG was evenly distributed over time, and the motor control pattern was consistent over both arms.

Most of this research focuses on the relationship between performance strategy and muscle activity with few studies exploring the perceptual properties of percussive sounds. One study investigated whether virtual and perceived sounds differed by the type of drum stroke, either normal or controlled (Dahl and Altenmuller, 2008). Various vectors (i.e., peak force, contact duration, and stick velocity) differed. Results showed that the participants produced a significantly higher peak force and shorter contact duration in the controlled stroke condition. Stick velocity, also associated with the controlled stroke, resulted in greater difference between pre- and postsurface impact. Sounds produced using controlled strokes were perceived as having a less full timbre and softer attack, indicating that different types of drum strokes induce significantly different levels of perception of drumming sounds. Another study analyzed sEMG signals associated with different instrument grasping patterns and varying tempo of hand percussion playing (Chong et al., 2015). When three different hand percussion instruments were compared, the small sphere type instrument elicited significantly greater muscle activation. In addition to the type of instrument, the results showed that tempo was also an important factor influencing degree of muscle activation. Although the above-mentioned studies provide detailed information regarding muscle movements in relation to playing techniques and methods, the interpretation of the results is limited to the specific musical strategies used in these studies. While muscle movements associated with classical music are well documented, those related to other music genres remain less understood.

Muscle activities measured by sEMG during drum playing have been widely studied from the trunk to the fingers; however, the information regarding muscle activation of the biceps brachii and triceps brachii during drum playing is still the blind spot for related academic areas, including rehabilitation and therapeutic use of instrument playing. Even though biceps brachii and triceps brachii are the main muscles in the upper arm, which would be activated by instrument playing for rehabilitation, little scientific evidence exists on their level of activity and relationship to sound intensity in drum playing. Thus, this study investigated sound intensity and muscle activation during drum playing.

\section{MATERIALS AND METHODS}

\section{Ethics statement}

Ethical approval was granted by the Ewha Womans University Institutional Review Board (approval number: 2012-07-03). The participants were informed of the procedure and provided written informed consent in accordance with the Declaration of Helsinki.

\section{Participants}

A total of 40 college students participated from universities located in central and remote areas of the Republic of Korea. All participants were nonmusic majors and had no history of muscle disease or current illness. The participants' demographic characteristics are presented in Table 1.

\section{EMG signal acquisition and data processing}

The 8-channel wireless QEMG-8 model (Laxtha Inc., Daejeon, Korea) was used to collect sEMG data when muscles were activated. The sEMG raw signals were measured at a sampling rate of $1,024 \mathrm{~Hz}$ and preamplified with a gain of 244. The amplified raw data were converted through an analog-to-digital converter with

Table 1. Demographic characteristics $(n=40)$

\begin{tabular}{lc}
\hline Variable & Value \\
\hline Gender, male:female & $22: 18$ \\
Age $(\mathrm{yr})$ & $21.3 \pm 1.8$ \\
Height $(\mathrm{cm})$ & $169.5 \pm 9.5$ \\
Weight $(\mathrm{kg})$ & $63.5 \pm 10.8$ \\
Body mass index $\left(\mathrm{kg} / \mathrm{m}^{2}\right)$ & $22.0 \pm 2.3$ \\
\hline
\end{tabular}

Values are presented as number or mean \pm standard deviation. 
12-bit resolution and recorded through Telescan ver. 2.91 (Laxtha Inc.) for data analysis. In this study, four channels were used to record sEMG signals from the biceps brachii and triceps brachii of the participant's dominant arm.

For data analysis, the digitized raw sEMG data were stored on a personal computer using Telescan ver. 2.91 and band-pass FFT filtered from $10 \mathrm{~Hz}$ to $450 \mathrm{~Hz}$ with the offset value removed. The root mean squares (RMS) of sEMG amplitude for the biceps brachii and triceps brachii were calculated with a moving window of $0.117 \mathrm{sec}$. MVC, which was induced while producing maximum force during striking motion for each muscle, was also obtained to normalize sEMG data. Then the percentage MVC value for the averaged RMS value was analyzed to determine differences in sEMG signals among the three stroke conditions.

\section{Electrode placement}

Disposable metal-plate electrodes with electrolyte gel coated $\mathrm{Ag} / \mathrm{AgCl}$ disks (Ag-AgCl, 3M Inc., Hamden, CT, USA) were used to measure sEMG signals of the biceps brachii and triceps brachii of each participant's dominant upper arm. The area on each participant's skin where the electrode was placed with an interelectrode distance of about $20 \mathrm{~mm}$ was cleaned with alcohol to reduce skin resistance to less than $5 \mathrm{k} \Omega$. A pair of electrodes was placed longitudinally on the belly of the biceps brachii and on the medial portion of the triceps brachii. The ground electrode was affixed to the back of the neck for electrical noise reduction. All the cables used for this study were shielded to eliminate electromagnetic interference and tied properly to the arms to prevent displacement of electrodes and to remove artifacts when playing the drum.

\section{Acoustic and audio analyzer}

The XL2 analyzer (NTi Audio, Liechtenstein, Switzerland) is a sound-level meter that monitors and records live sound events and/or environmental noise. The XL2 with the M2230 microphone (NTi Audio) has the capability of correcting values between the loudest point of a live event and the measurement positions in accordance with DIN 15905-5 and SLV. In parallel with the wide band parameters, the XL2 measures the real-time spectrum either in $1 / 1$ or $1 / 3$ octave-band resolution.

\section{Drum}

A snare drum (Ludwig, $36 \times 12.7 \mathrm{~cm}$ ) was used, and its wire was removed to produce tom-tom sounds. The drumstick used to play the drum was standard (Preminum 7A) with a length of 39 $\mathrm{cm}$ and a diameter of $1.4 \mathrm{~cm}$.

\section{Experiment setup}

EMG signals were recorded from the biceps brachii and triceps brachii of each participant's dominant upper arm in an acoustic anechoic chamber. Participants sat down on a chair facing the drum. Participants practiced playing the drum 5 times to get familiar with how strong to stroke in order to produce the three different levels of stroke (soft, medium, strong). Soft stroke, medium stroke, and strong stroke were defined by their decibel range as measured by the XL2 audio analyzer. Soft stroke ranged from 94 to $96 \mathrm{~dB}$, medium stroke from 101 to $103 \mathrm{~dB}$, and strong stroke from 108 to $110 \mathrm{~dB}$. The examiner confirmed the participants' posture and appropriate stroke level (Fig. 1).

The participants were instructed to strike the drum with the drumstick 10 times for each stroke condition, for a total of 30 hits. Each drum strike was preceded by a visual cue generated at 3 -sec intervals. There was a 10 -sec break between sound conditions to refresh the muscles. Sound from the drum was muffled with soft lint to prepare for the next strike. These striking movements were repeated using the participant's dominant arm but without the drumstick.

Drum playing can make various sounds and be generated from a variety of muscles such as the shoulder, upper arm, forearm, wrist, and fingers. However, to obtain data related to the flexion and extension of the upper arm to move the forearm for the purpose of rehabilitation, it is important to exclude any possible mus-

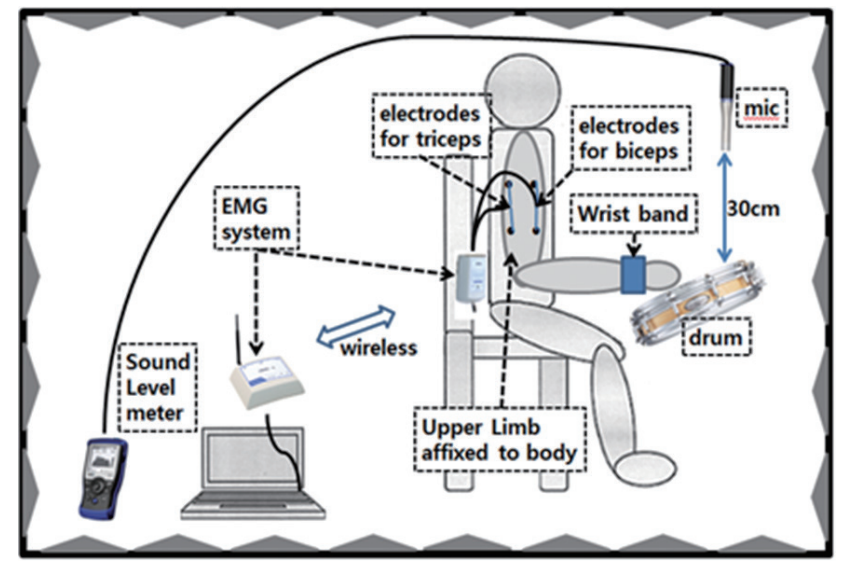

Fig. 1. Experimental setup for the study. A microphone (M2230, NTi Audio, Liechtenstein, Switzerland) extended from an acoustic and audio analyzer (XL2, NTi Audio) was placed $30 \mathrm{~cm}$ above the surface of the drum. The loudness of the drum sounds and the strength of the muscle activations for each participant were measured throughout the three stroke conditions both with and without a drumstick. EMG, electromyography. 
cle movements other than from the biceps brachii and triceps brachii. To this end, a wrist brace was used to prevent a wrist snap from making any additional sound, and the upper arm was affixed to the side of the body to stop shoulder movement from being involved in the striking of the drum. A microphone (M2230) connected to an acoustic and audio analyzer (XL2) was placed $30 \mathrm{~cm}$ above the surface of the drum. The loudness of the drum sound and the strength of the muscle activations for each participant were measured for each condition.

\section{Statistical analysis}

Once participants completed the given task, the researcher saved the decibel sound pressure level and sEMG data in Volt. RMS value of sEMG data was selected and averaged before and after around $0.5 \mathrm{sec}$ of each hit depending on the speed of each participant's drum strikes, and decibel data were selected from the target hit (LEQmax) for further statistical analysis. The selected data were analyzed using a two-way repeated measures analysis of variance (ANOVA). The statistical tool used was IBM SPSS Statistics ver. 20.0 (IBM Co., Armonk, NY, USA).

\section{RESULTS}

\section{Muscle activation by level of stroke and drumstick use}

For biceps brachii and triceps brachii, the muscle activation values were measured during striking of the drum with the use of the drumstick versus without the use of the drumstick for three different levels of stroke: soft, medium, and strong. With regard to muscle activation of biceps brachii, the highest muscle activa-

Table 2. sEMG MVC (\%) data depending on the sound intensity $(n=40)$

\begin{tabular}{lllc}
\hline Muscle & Drumstick use & Level of stroke & sEMG MVC (\%) \\
\hline Biceps & Use & Soft & $31.9 \pm 23.0$ \\
& & Medium & $40.8 \pm 15.3$ \\
& Strong & $51.6 \pm 42.2$ \\
& No use & Soft & $24.5 \pm 15.3$ \\
& & Medium & $34.5 \pm 30.9$ \\
Triceps & Strong & $53.3 \pm 43.4$ \\
& Use & Soft & $15.2 \pm 15.1$ \\
& & Medium & $18.4 \pm 18.1$ \\
& Strong & $35.5 \pm 44.9$ \\
& No use & Soft & $22.4 \pm 20.9$ \\
& & Medium & $34.2 \pm 31.6$ \\
& & Strong & $57.9 \pm 40.4$ \\
\hline
\end{tabular}

Values are presented as mean \pm standard deviation. sEMG MVC, surface electromyography maximal voluntary contraction. tion was observed in the condition of strong stroke regardless of whether the drumstick was used or not, followed by the medium and soft conditions, respectively. While greater sEMG values were observed for the biceps brachii when playing with a drumstick than when playing without the drumstick for the soft and medium stroke conditions, strong drum playing elicited greater muscle activation under the no drumstick condition. In terms of muscle activation of the triceps brachii, the highest sEMG data were observed for the strong stroke condition both with and without the drumstick. In each stroke condition, drum playing by hand led to higher muscle activation of the triceps brachii than using a drumstick. The sEMG data for each condition are summarized in Table 2.

A two-way repeated measures ANOVA showed that for biceps brachii, the main effect for stroke condition reached statistical significance, $F(2,78)=26.87, P<0.01$, while there were no significant differences in sound intensity depending on the use of the drumstick, $F(1,39)=0.655, P=0.423$. There was also a significant interaction between stroke and drumstick use, $F(2,78)=4.786$, $P=0.011$ (Fig. 2). A post hoc analysis with Bonferroni correction showed that significant differences in the sound intensity were found between soft and medium strokes $(P<0.01)$ and between medium and strong strokes $(P<0.01)$.

With regard to the triceps brachii, when playing the drum both with the drumstick and without the drumstick, the highest muscle activation was elicited in the strong stroke condition, followed by medium and soft stroke, respectively. When playing the drum without the drumstick, greater muscle activation was generated in all three stroke conditions, compared to when using a drumstick (Table 2). A two-way repeated measures ANOVA showed that the main effect of stroke condition reached statistical significance, $F$ $(2,78)=33.24, P<0.01$, and the main effect of drumstick use was also statistically significant, $F(1,39)=13.28, P=0.001$. There was also a significant interaction between stroke and drumstick

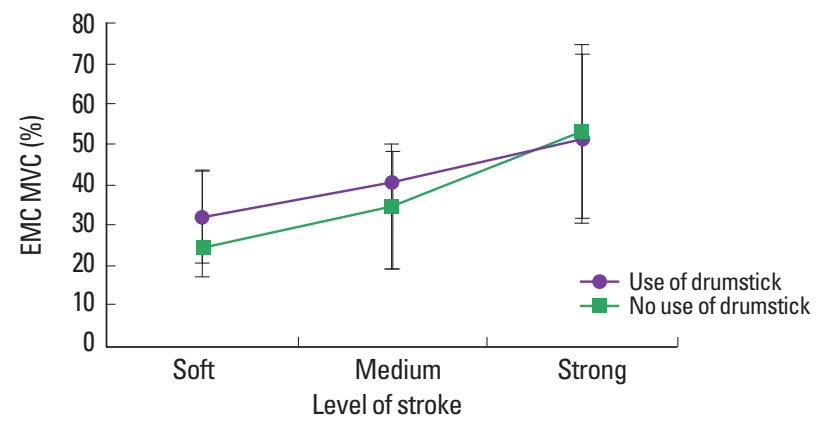

Fig. 2. Electromyography maximal voluntary contraction (EMG MVC) (\%) depending on the stroke and drumstick use for biceps brachii. 


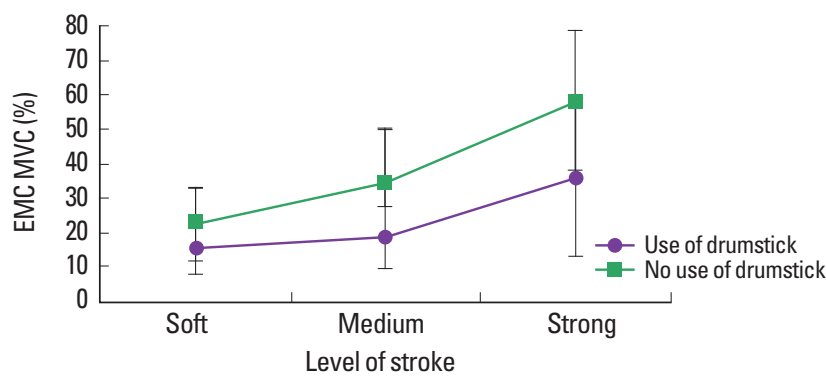

Fig. 3. Electromyography maximal voluntary contraction (EMG MVC) (\%) depending on the stroke and drumstick use for triceps brachii.

Table 3. Sound intensity level (dB) depending on the level of stroke and drumstick use $(n=40)$

\begin{tabular}{lcc}
\hline Drumstick use & Level of stroke & Sound intensity level (dB) \\
\hline Use & Soft & $95.4 \pm 4.9$ \\
& Medium & $102.0 \pm 5.4$ \\
No use & Strong & $108.6 \pm 6.3$ \\
& Soft & $94.0 \pm 5.8$ \\
& Medium & $101.3 \pm 6.4$ \\
& Strong & $108.6 \pm 7.5$ \\
\hline
\end{tabular}

Values are presented as mean \pm standard deviation.

use, $F(2,78)=6.51, P=0.002$ (Fig. 3). A post boc analysis with Bonferroni correction showed that significant differences in the sound intensity were found between soft and medium strokes $(P<0.01)$ and between medium and strong strokes $(P<0.01)$. Significantly greater muscle activation was measured when playing the drum without a drumstick compared to when using a drumstick $(P=0.001)$.

\section{Produced sound intensity level depending on the level of stroke}

When striking the drum with and without the drumstick in each of the three levels of stroke (i.e., soft, medium, and strong), the produced sound intensity $(\mathrm{dB})$ was measured. For each stroke condition, similar levels of sound intensity were measured when playing the drum with a drumstick and without a drumstick (Table 3). The highest sound intensity was observed in the condition of strong stroke, followed by medium and soft stroke, respectively. A two-way repeated measures ANOVA showed that the main effect of stroke condition reached statistical significance, $F(2,78)$ $=259.77, P<0.01$, while there were no significant differences in the sound intensity level depending on the use of drumstick, $F$ (1, $39)=1.13, P=0.294$. There was also a significant interaction between stroke and drumstick use, $F(2,78)=3.73, P=0.028$ (Fig. 4). A post hoc analysis with Bonferroni correction showed that significant differences in the sound intensity were found between soft

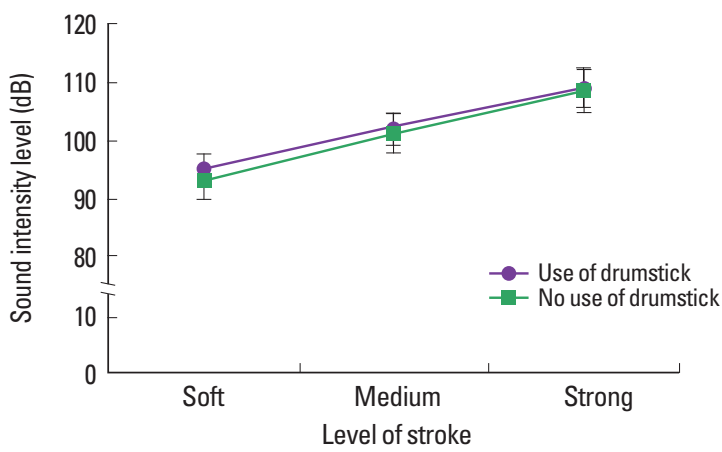

Fig. 4. Sound intensity level (dB) depending on stroke and drumstick use.

and medium stroke $(P<0.01)$ and between medium and strong stroke $(P<0.01)$.

\section{DISCUSSION}

In the present study, we measured the amplitude of sEMG during striking motion involving extension and flexion of the dominant upper arm. Regarding the use of a drumstick, muscle activation of the biceps brachii was larger for soft and medium drum playing with the drumstick while the triceps brachii showed smaller activation for all three stroke conditions when playing with the drumstick. This is because drum striking is made mainly by the downward movement of the forearm, which involves the action of elbow extension, generated by the triceps brachii. In this movement, the biceps brachii mainly acts on controlling speed and direction against the movement of the triceps brachii.

Regardless of drumstick use, similar sound intensity levels were demonstrated in each striking condition. This finding suggests that the drumstick is not necessarily required to control the produced sound; however, less vibrotactile feedback is transmitted to the hand when using a drumstick. As revealed in Chong et al. (2015), manipulating tempo during instrument playing has a greater impact than adjusting sound intensity level on increasing muscle activation. Also, the difference in sEMG of the triceps brachii between sound levels was larger than that of the biceps brachii. This observation coincides with results from Fujisawa and Miura (2010) who examined how wrist muscle activity differed by drumstick size. The authors found that EMG activity was significantly greater with decreased length and increased weight of the drumstick. For upper limb rehabilitation purposes, drum playing with a drumstick can be an effective option depending on the target muscle and the need for real-time vibrotactile feedback.

A possible use of the data from this study is in designing physi- 
cal rehabilitation programs using drum playing. Alternating the produced sound level $(\mathrm{dB})$ while playing a drum can be a practical intervention, and more effective therapeutic instrument playing can be provided if the required sound intensity level is controlled. It was generally believed that using drumsticks during drum playing involved more motor control since grabbing the drumstick was required; therefore, more muscle activation was expected. However, this study demonstrated via sEMG data that muscle activation is actually not associated with the use of drumsticks in drum playing. Thus, the use of drumsticks results in less movement being required and in a relatively reduced range of motion. The wider range of motion resulting from drum playing without drumsticks produces higher muscle activation making drum playing an ideal therapeutic treatment with the drum placed so that it addresses the upper limb targeted for rehabilitation.

In sum, the sound level should be loud enough to induce a certain level of muscle activation, and not using drumsticks is recommended when the therapist wants to focus on the triceps brachii muscle group. Also, hand drumming should be considered to increase muscle activation of the biceps brachii muscle group in the upper arms. Based on the results of this study, future research should investigate muscle activation and instrument playing in individuals with physical disabilities.

\section{CONFLICT OF INTEREST}

No potential conflict of interest relevant to this article was reported.

\section{ACKNOWLEDGMENTS}

This work was supported in part by Basic Science Research Program through the National Research Foundation of Korea (NRF) funded by the Ministry of Science, ICT and Future Plan- ning (NRF-2013-R1A1A1013430) and in part by the National Research Foundation of Korea Grant funded by the Korean Government (NRF-2012-1788-001-2) and in part by the Soonchunhyang University Research Fund.

\section{REFERENCES}

Chong HJ, Kim SJ, Lee EK, Yoo GE. Analysis of surface EMG activation in hand percussion playing depending on the grasping type and the tempo. J Exerc Rehabil 2015;11:228-235.

Dahl S, Altenmuller E. Motor control in drumming: influence of movement pattern on contact force and sound characteristics. J Acoust Soc Am 2008;123:3122-3127.

Fujii S, Kudo K, Ohtsuki T, Oda S. Tapping performance and underlying wrist muscle activity of non-drummers, drummers, and the world's fastest drummer. Neurosci Lett 2009;459:69-73.

Fujisawa T, Miura M. Investigating a playing strategy for drumming using surface electromyograms. Acoust Sci Technol 2010;31:300-303.

Guettler K. Electromyography and muscle activities in double bass playing. Music Percept 1992;9:303-309.

Jeong E. A rationale for instrumental music playing for upper extremity rehabilitation in subacute stroke. J Music Hum Behav 2013;10:1-23.

Lai CJ, Chan RC, Yang TF, Penn IW. EMG changes during graded isometric exercise in pianists: comparison with non-musicians. J Chin Med Assoc 2008;71:571-575.

Oikawa N, Tsubota S, Chikenji T, Chin G, Aoki M. Wrist positioning and muscle activities in the wrist extensor and flexor during piano playing. Hong Kong J Occup Ther 2011;21:41-46.

Penn IW, Chuang TY, Chan RC, Hsu TC. EMG power spectrum analysis of first dorsal interosseous muscle in pianists. Med Sci Sports Exerc 1999;31:1834-1838.

Thiem B, Greene D, Prassas S, Thaut M. Left arm muscle activation and movement patterns in cellists employing a playing technique using rhythmic cuing. Med Probl Perform Artist 1994;9:89-96. 